\title{
Down-regulation of MRPS23 inhibits rat breast cancer proliferation and metastasis
}

\author{
Yan Gao ${ }^{1, *}$, Fuyan Li ${ }^{1, *}$, Hong Zhou ${ }^{1}$, Yi Yang $^{1}$, Ruimin Wu${ }^{1}$, Yijia Chen ${ }^{1}$, Wei $\mathbf{L i}^{1}$, \\ Yang $\mathrm{Li}^{1}$, Xueqin $\mathrm{Xu}^{1}$, Changbin $\mathrm{Ke}^{1}$ and Zhijun $\mathrm{Pei}^{1}$ \\ ${ }^{1}$ Department of PET Center and Institute of Anesthesiology and Pain, Taihe Hospital, Hubei University of medicine, Hubei, \\ China \\ "These authors contributed equally to this work
}

Correspondence to: Zhijun Pei, email: pzjun1980@yeah.net

Changbin Ke, email: changbinke@gmail.com

Keywords: MRPS23, breast cancer, metastasis, p53, p21 WAF1/CIP1

Received: October 31, $2016 \quad$ Accepted: April 28, $2017 \quad$ Published: May 15, 2017

Copyright: Gao et al. This is an open-access article distributed under the terms of the Creative Commons Attribution License 3.0 (CC BY

3.0), which permits unrestricted use, distribution, and reproduction in any medium, provided the original author and source are credited.

\section{ABSTRACT}

Mitochondrial ribosomal protein S23 (MRPS23) has been shown to be involved in breast cancer cell proliferation and metastatic phenotypes of cervical cancer. Here we investigated its biological features in breast cancer for the first time. It demonstrated that knockdown of MRPS23 reduced breast cancer cell proliferation and induced apoptosis in vitro. Besides, shRNA targeting MRPS23 (shMRPS23) inhibited tumour proliferation and metastasis by blocking tumor angiogenesis in breast cancer xenograft rat model. Small animal positron emission tomography/computed tomography (PET/CT) with 2'-deoxy-2'$\left[{ }^{18} \mathrm{~F}\right]$ fluoro-D-glucose (FDG) was performed at four weeks after tumour cell injection. We found that FDG maximum standardized uptake value (SUVmax) significantly decreased by $31 \pm 3 \%$ in the shMRPS23-treated group. But this change was not independent of metabolic tumour size. In addition, we also found that shMRPS23 could significantly suppress breast cancer metastasis through inhibiting epithelial mesenchymal transition (EMT) phenotype. The epithelial marker E-cadherin was increased, whereas the metastasis associated gene vimentin was decreased. Mechanistically, shMRPS23-treated tumours failed to progress through $\mathrm{p} 53$ and p21 ${ }^{\text {WAF1/CIP1 }}$ activation, but not cytochrome c-mediated pathway. These findings suggest that MRPS23 is a potential therapeutic target for interference of breast cancer proliferation, angiogenesis and metastasis.

\section{INTRODUCTION}

Breast cancer is a major cause of cancer-related death, with about 1.2 million newly diagnosed cases every year and increasing numbers of cases in developing countries, including China $[1,2]$. The reasons for high risk and mortality rate is that it can proliferate and metastasize in the lung, lymph node or elsewhere [3], especially in bone metastasis [4]. Although much effort is being spent on developing new therapeutic targets, the molecular mechanisms of breast cancer tumourigenesis and metastasis is still unclear, and the overall survival rate is still low as a consequence.

This study focused on a $22 \mathrm{kDa}$ mitochondrial ribosomal protein S23 (MRPS23) that is located at 17q22-23.
MRPS23 was reported to be overexpressed in various cancers, such as breast [5, 6], ovarian [7], colorectal [8], uterine leiomyoma [9] and hepatocellular carcinoma [10]. Furthermore, it has been known as a key genetic drivers that is essential for cell proliferation and is uniquely expressed in breast cancer patients [5]. MRPS23 is also more strongly associated with metastatic phenotypes in cervical cancer $[11,12]$. These studies indicate that MRPS23 may exhibit a negative impact on proliferative and metastatic breast cancer. Nevertheless, the exact function of the molecule and the mechanism by which MRPS23 inhibits the proliferation and metastasis of breast cancer remain unknown.

Recent studies have examined the biological functions and the possible mechanisms of mitochondrial ribosomal proteins (MRPs) in cancers individually. Several evidence 
have shown that MRPs are involved in cell proliferation and mitochondrial apoptosis through the $\mathrm{p} 21^{\mathrm{WAF} 1 / \mathrm{CIP} 1}$ [13], p53 [14-16] and cytochrome c (Cyt c) -mediated pathway [17]. $\mathrm{p} 21^{\mathrm{WAF} 1 / \mathrm{CIP} 1}$ may be regulated through either p53-dependent [18] or p53-independent mechanism [19]. However, for the new target gene MRPS23, there are few reports as to whether this mechanism occurs. Angiogenesis, is a key process in pathological conditions that drives the progression of tumour [20]. In addition, epithelial mesenchymal transition (EMT) plays an important role in the metastatic process of breast cancer cells, of which the E-cadherin and vimentin proteins are key components [21]. Here, we investigated the expression and biological significance of MRPS23 in breast cancer, and demonstrated that shMRPS23 inhibits cell proliferation and survival by repressing p53 and p21 ${ }^{\mathrm{WAF} 1 / \mathrm{CIP} 1}$. We further described a critical role for MRPS23 as an important suppressor of proliferation and metastasis using ${ }^{18}$ F-FDG PET imaging.

\section{RESULTS}

\section{Downregulation of MRPS23 inhibited breast cancer proliferation and induced apoptosis in vitro}

To validate the efficiency of shMRPS23 knockdown and obtain an appropriate multiplicity of infection (MOI), we examined the expression of MRPS23 in Walker256 cells that were successfully infected with LVshMRPS23 vectors at a MOI of 1,5 and 10 respectively. The subcellular fractionation experiments using confocal immunofluorescent microscopy showed that MRPS23 (blue) was localized in the mitochondria (red) and nuclear in Walker256 cells (Figure 1A). Compared with the LV-shCtrl and PBS control groups, LV-shMRPS23 significantly inhibited MRPS23 mRNA and protein expression in cell culture $(p<0.05)$ (Figure 1B) at a MOI of 5. MTS experiments showed that knockdown of MRPS23 inhibited breast cancer cell proliferation in vitro compared to controls (Figure 1C). The infection rate was about $80-90 \%$, but TUNEL-positive cells were more frequently observed $48 \mathrm{~h}$ post transfection with shMRPS23 (Figure 1E). Quantitative analyses revealed that the cellular apoptosis rate was about $4 \%$ in Control, shCtrl and $13.2 \%$ in shMRPS23 groups $(p<0.05)$ (Figure 1D).

\section{shMRPS23 inhibited breast cancer proliferation and angiogenesis in vivo}

To further study the biological function of MRPS23 in breast cancer progression in vivo, we first investigated the expression of MRPS23 in breast tumour tissues. Immunohistochemistry revealed that MRPS23 is strongly expressed in tumour tissues compared with adjacent nontumour tissue (Figure 2A). The colocalization of MRPS23 with LV depicted the inhibited expression of MRPS23 in shMRPS23-treated tumour (Figure 2B). Knockdown of MRPS23 also inhibited the protein and mRNA expression of MRPS23 in shMRPS23-treated tumours, compared with Control and shCtrl groups $(p<0.05)$ (Figure 2C). To illustrate whether the decreased MRPS23 contributes to breast cancer cell proliferation and angiogenesis, we performed ki-67 and CD34 stain, respectively. The proliferation index is typically $72 \%-78 \%$ for Control group, 64\%-70\% for shCtrl tumour, and 38\%-40\% for shMRPS23 tumour (Figure 2D). Moreover, CD34 was concentrated at the tumour- adjacent mucosa sites (Figure 2E). There was a significant difference in mean MVD $(P<0.05)$ between shMRPS23-related tumour and control tumour. Taken together, these data suggested a suppressive role of shMRPS23 in breast cancer proliferation and angiogenesis in vivo.

\section{MRPS23 knockdown suppressed breast cancer progression by upregulating p53 and p21 ${ }^{\mathrm{WAF} 1 / \mathrm{CIP} 1}$}

Given the critical role of MRPs on differential regulation of p53, p2 $1^{\mathrm{WAF} 1 / \mathrm{CIP} 1}$ and Cyt c levels in tumourigenesis, we wondered whether MRPS23 was associated with this signalling pathway. As expected, shMRPS23 colocalized with p53 (Figure 3A), and the mRNA and protein expression levels of p53 dramatically increased by $1.5-4$ fold in shMRPS23-treated tumours $(p<0.05)$ (Figure 3B). Concomitant with the induction of $\mathrm{p} 53$, treatment with shMRPS23 significantly increased the level of $\mathrm{p} 21^{\mathrm{WAF} 1 / \mathrm{CIP} 1}$ protein $(p<0.05)$ (Figure 3B). However, this was not accompanied by an increase in $\mathrm{p} 21$ ${ }_{\text {WAF } 1 / C I P 1}$ mRNA. In addition, no differences were found in Cyt C mRNA expression. Knockdown of MRPS23 did not trigger the release of Cyt C (Figure 3B) in control and shMRPS23-treated conditions. These observations suggest that depletion of MRPS23 induced the expression of p53 and $\mathrm{p} 21^{\mathrm{WAF} 1 / \mathrm{CIP} 1}$ protein.

\section{shMRPS23 reduced the relative tumour metabolism and inhibited tumour metastasis as detected by ${ }^{18}$ F-FDG microPET imaging}

To investigate the effect of shMRPS23 treatment in our breast cancer model, we performed ${ }^{18} \mathrm{~F}$-FDG PET/CT imaging. Representative images are shown in Figure 4A, VOIs were drawn to first determine metabolic volume of lesion (Figure 4B) and then estimate focal FDG uptake using SUVmax and SUVmean (Figure 4C). We observed apparent bone destruction in the right hindleg compared to the contralateral side. Importantly, increased FDG uptake in the centre of tumour focus was observed in the nontreated rats, whereas liquefactive necrosis was seen in the shMRPS23-treated rat (yellow arrow). The metabolic volume of VOIs steadily decreased in shMRPS23, 
yet not significantly. Notably, there was no significant difference between the Control and shCtrl group in terms of SUV elevations. Nevertheless, there was lower FDG uptake observed in the shMRPS23 treatment group, as indicated by a 1.5-fold decrease in SUVmax and a 1.45-fold decrease in SUVmean compared with Control group. Figure 4 depicts inguinal lymph node metastasis in rats infected with PBS (red arrow) and peritoneum metastasis in the shCtrl group. However, only one of six rats in shMRPS23 treatment group showed lymph nodes metastasis. Collectively, these data provided strong evidence that shMRPS23 reduces the relative tumour metabolism and inhibits tumour metastasis in the course of tumour progression.

\section{MRPS23 silencing on breast cancer metastasis via disturbing EMT}

Importantly, our findings indicated that shMRPS23 impaired metastasis in vivo (Figure 4A). To gain additional mechanistic insight into the role of MRPS23 in the metastasis cascade, we focus on the epithelial marker
E-cadherin and mesenchymal marker vimentin, which play a major role in the EMT process that contributes to tumour progression [22]. As is shown in Figure 5A, E-cadherin was localized mostly to the membrane of the leading edge in shMRPS23-treated tumours, whereas staining was diffuse to the surrounding tissue in the control groups. Conversely, vimentin expression was repressed (Figure 5B) in MRPS23 knockdown tumours compared with shCtrl-infected samples. Western analyses revealed that global levels of E-cadherin were substantially higher in the MRPS23-deficient group than in the control, whereas vimentin showed the opposite pattern (Figure 5C), as evidenced by mRNA analysis at the same time. The results suggest that MRPS23 inhibited the metastasis properties of breast cancer through influencing of EMT.

\section{DISCUSSION}

Present genomic data sets have demonstrated that MRPS23 is upregulated in a series of cancers and it plays an essential role in breast cancer cell proliferation [5] and cervical metastasis [12]. These findings suggest that

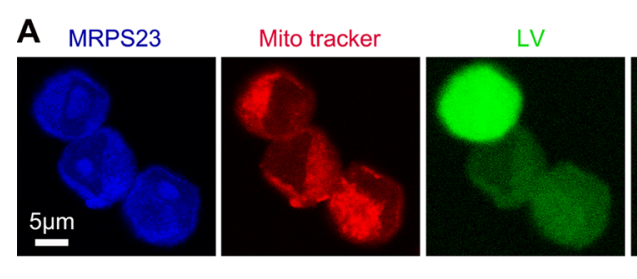

B
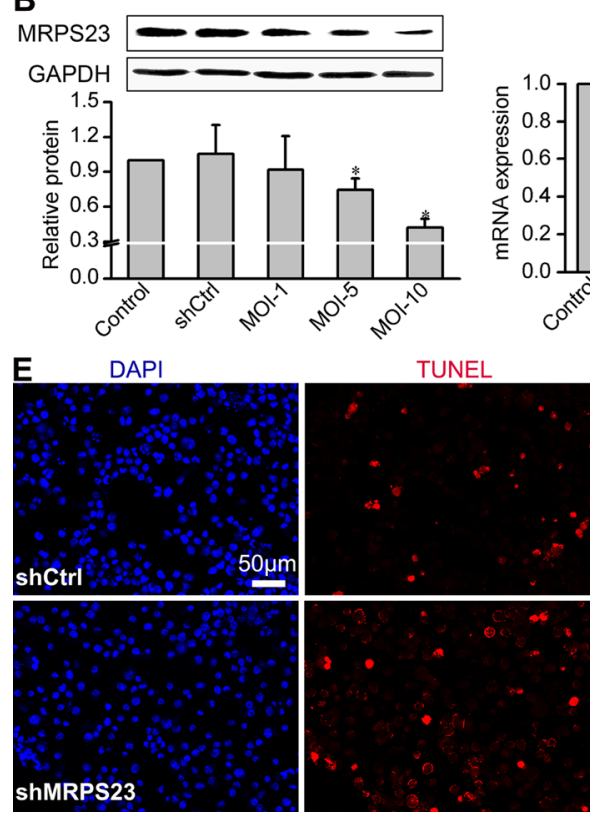

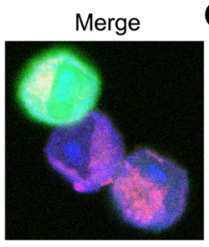

D
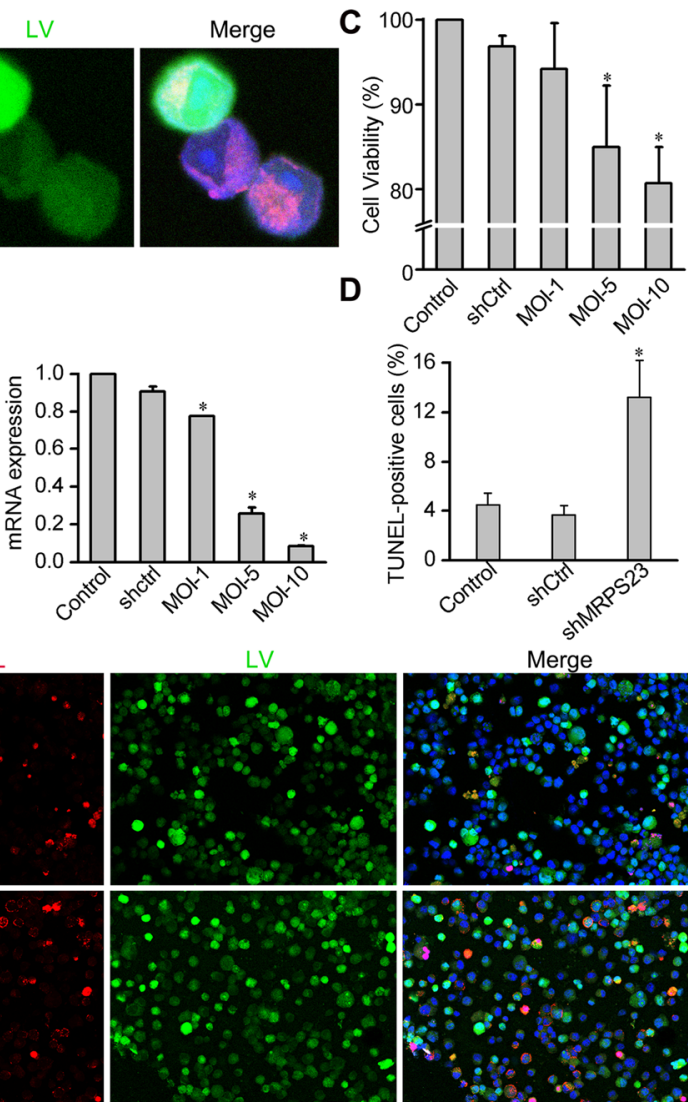

Figure 1: Downregulation of MRPS23 reduces Walker256 carcinoma cell viability and induces apoptosis. (A) Representative images of subcellular localization of MRPS23 by co-staining with $100 \mathrm{nM}$ MitoTracker Red (pseudo-coloured red) and anti-MRPS23 (pseudo-coloured blue). Walker 256 cancer cells was infected with LV-shMRPS23. (B) Efficiency of LV-shRNA interference was detected by real-time PCR and western blotting. (C) Walker256 carcinoma cell viability was detected by MTS assay. (D) Quantification of the apoptotic cells was presented as the percentage of apoptotic cells. ( ${ }^{*} p<0.05$ vs. control condition). (E) Confocal imaging of LV (green fluorescent), TUNEL (red fluorescent) and DAPI (blue fluorescent) staining in Walker256 cells $48 \mathrm{~h}$ post transfection. 
MRPS23 is tumour promoter in breast cancer. To the best of our knowledge, this is the first study to demonstrate that longterm depletion of MRPS23 complex led to repressed breast tumour growth in the xenograft model. Downregulation of MRPS23 by LV-mediated MRPS23 shRNA suppressed breast cancer metastasis shown by ${ }^{18} \mathrm{~F}-\mathrm{FDG}$ microPET imaging. Moreover, shMRPS23 markedly reduced FDG uptake of breast cancer cells. In agreement with recent observations, an important finding is that the SUV value was not independent of tumour size. Our findings may supports an oncosuppressive role of MRPS23 shRNA in rat breast cancer proliferation, angiogenesis and metastasis.

However, the molecular mechanism of how breast cancer cell progression is suppresed by knockdown of MRPS23 remains to be illustrated. Mitochondrial ribosomal protein are known to be synthesized in the cytosol and then imported into mitochondria for assembly; they are responsible for translation of 13 mitochondrial
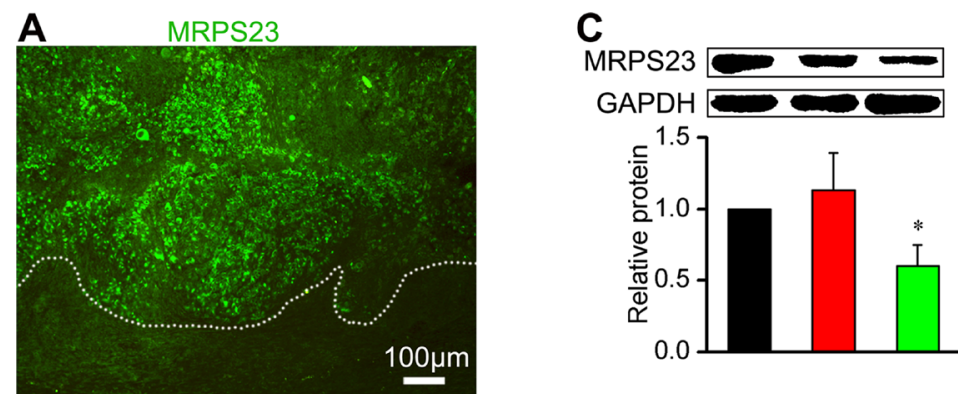

GAPDH

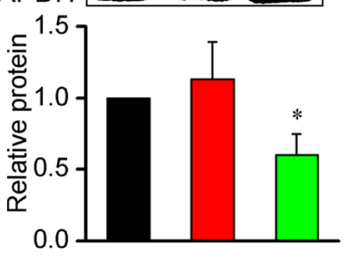

MRPS23+ LV
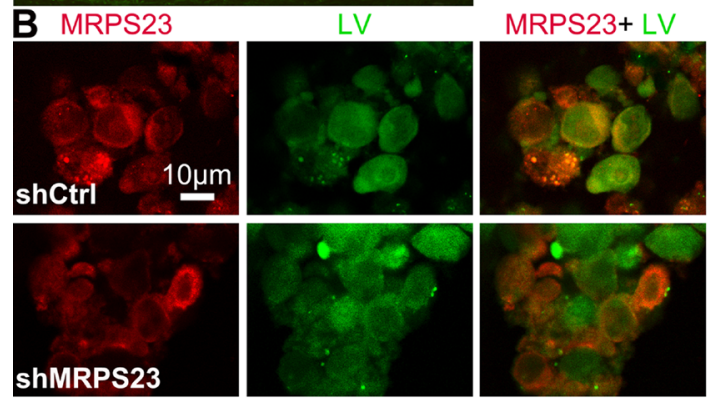

E
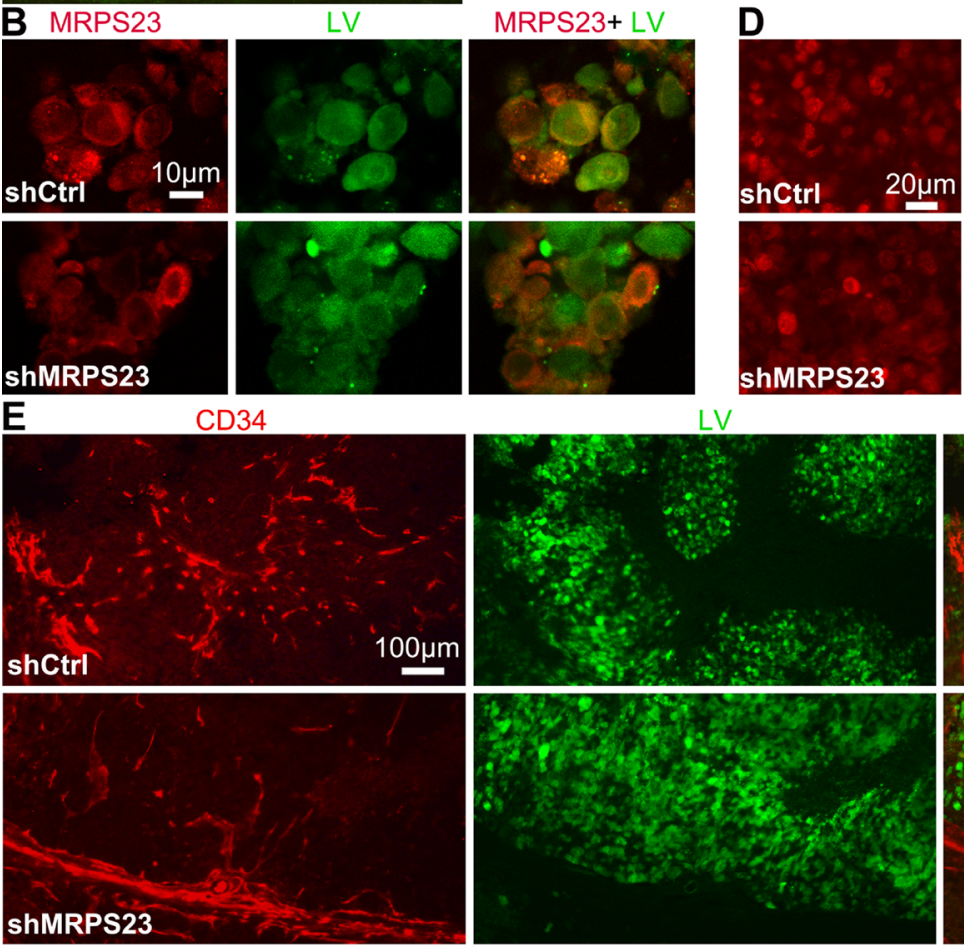

ShMRPS23

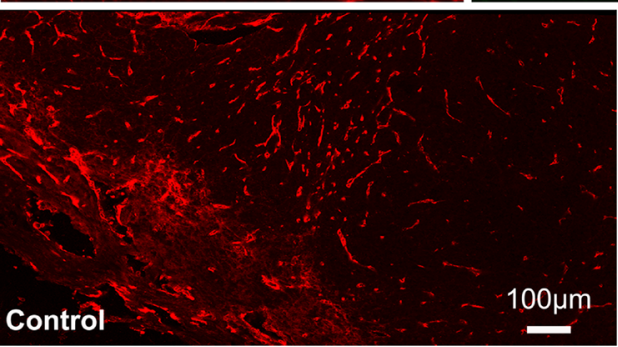

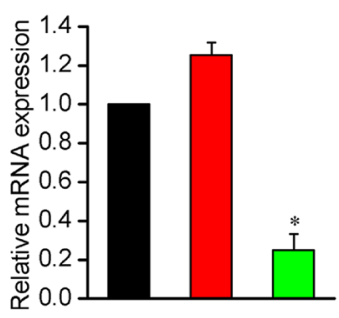

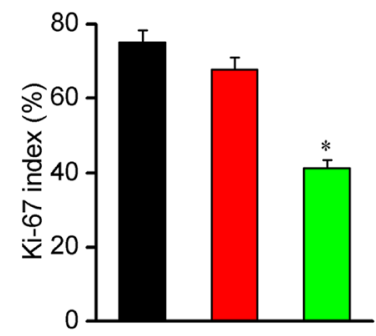

$\mathrm{LV}+\mathrm{CD} 34$

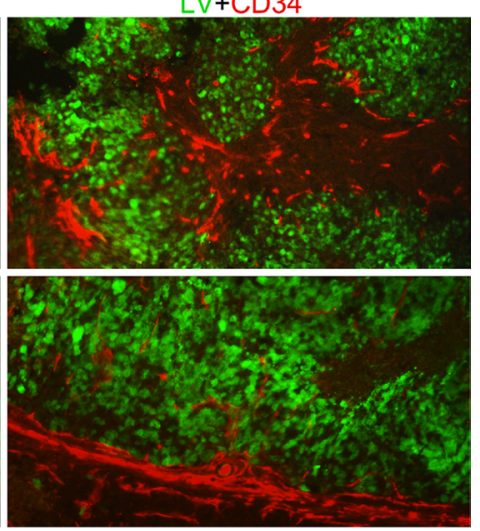

Control

shCtrl

shMRPS23

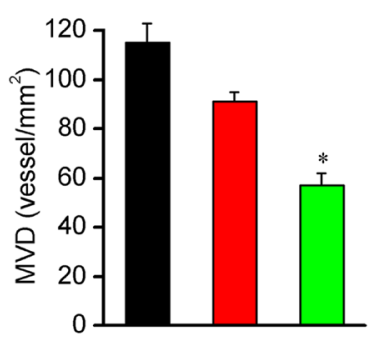

Figure 2: shMRPS23 inhibits cancer cell proliferation and angiogenesis in vivo. (A) MRPS23 strongly expressed in tumour tissues, the white dotted line is drawn to distinguish viable tumour from tumour necrosis. (B) Localization of MRPS23 (red) in shCtrl or shMRPS23 treated tumour. (C) MRPS23 was silenced in tumour cells by LV-shRNA in vivo. (D) Representative image of immunohistochemical staining of proliferation marker Ki-67 expression in each group and the percentage of Ki-67-positive cells (overlap between Ki67 and virus) in each field. (E) Characterization and images of vascular network by staining for CD34 and quantification of MVD (vessel $/ \mathrm{mm}^{2}$ ) in each group. 

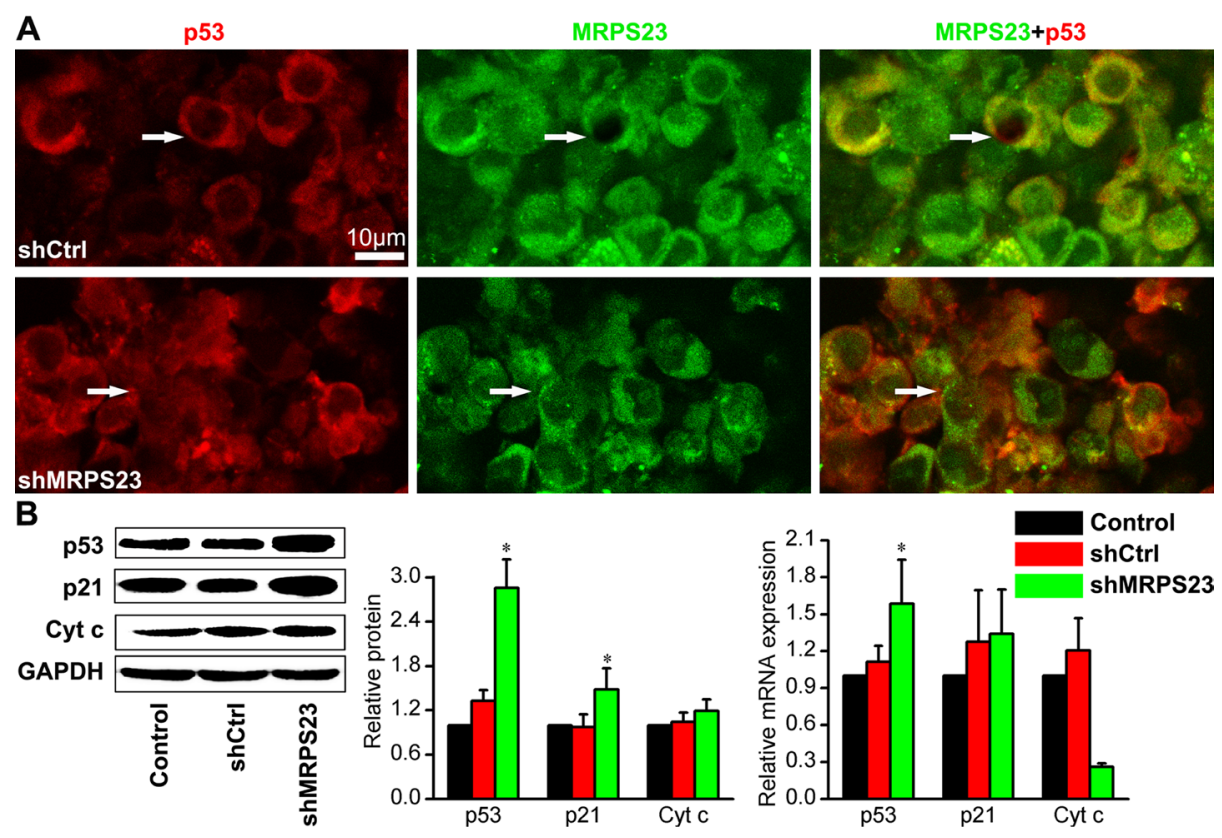

Figure 3: MRPS23 knockdown activates p53 and p21 WAF1/CIP1. (A) Immunofluorescence staining of tumor sections showing that p53 (pseudo-coloured red) expression increases while MRPS23 (pseudo-coloured green) expression decreases after LV-shMRPS23 transfection. (B) Western blot and qPCR analysis of p53 and p21 WAF1/CIP1 (p21) in the tumours of each group, showing the molecular effects induced after shMRPS23 treatment. ( ${ }^{*} p<0.05$ vs. control condition).
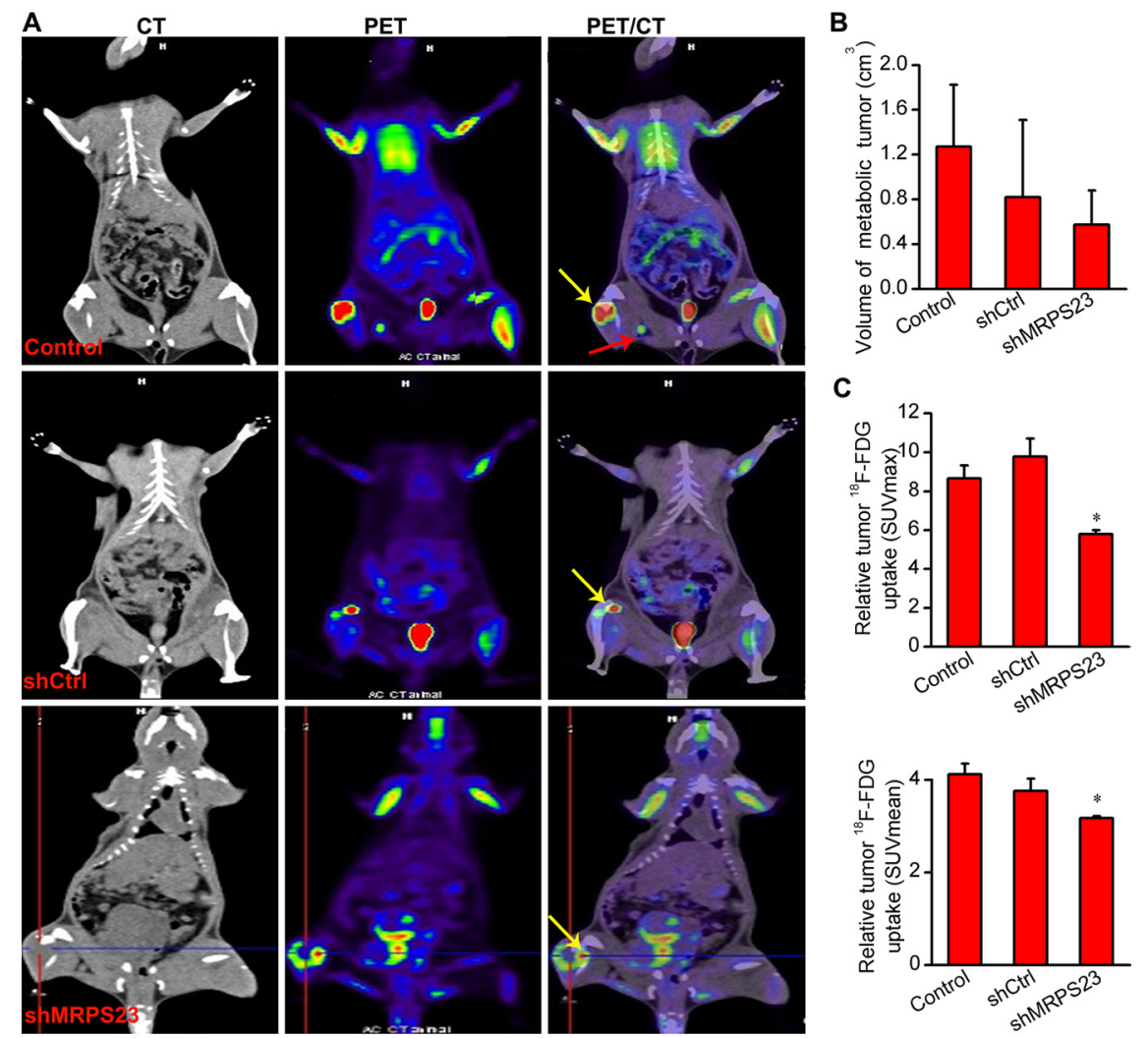

Figure 4: ${ }^{18}$ F-FDG PET analysis of tumour metabolism and metabolic tumour volume. (A) Co-registered PET images and CT images from SD rats with ${ }^{18} \mathrm{~F}$ - FDG injected after four weeks treatment with shMRPS23, $(n=4)$; yellow arrow depicts SUVmax sites and liquefactive necrosis (Criss Cross), red arrow indicates lymph nodes metastasis. (B, C) Quantification of metabolic volume and FDG uptake of tumours in the three experimental groups. The tumor uptake of FDG was markedly reduced in the shMRPS23-treated rat compared to the control groups $(* p<0.05$ vs. control condition). 
mRNAs [23]. The proliferation of cultured mammalian cells can be impaired following disruption of mitochondrial function [24]. If the expression of MRPS23 was inhibited, it could disturb the mitochondrial protein synthesis, affect function of mitochondrial and impair breast cancer cell proliferation. Several studies indicate that the ribosomal proteins and MRPs may be directly involved in the process of cancer proliferation and metastasis $[25,26]$, which are dependent on the stimulated activity of p53 and $\mathrm{p} 21^{\mathrm{WAF} 1 / \mathrm{CIP} 1}[16,27,28]$. Yoo et al. reported that the MRPL41 suppresses cell growth in association with $\mathrm{p} 53$, p2 $7^{\mathrm{Kip} 1}$ [15] and p21 ${ }^{\mathrm{WAF} 1 / \mathrm{CIP} 1}$ [29]. Chen et al. showed that MRPS36 delays cell progression in association with p53 modification and $\mathrm{p} 21^{\mathrm{WAF} 1 / \mathrm{CIP} 1}$ expression [14]. Interestingly, we found that silencing of MRPS23 inhibited proliferation and induced apoptosis by upregulating p53 and p21 WAF1/ ${ }^{\mathrm{CIP} 1}$ protein, but not $\mathrm{p} 21^{\mathrm{WAF} 1 / \mathrm{CIP} 1} \mathrm{mRNA}$. The role of $\mathrm{p} 53$ has been shown to be to extend even beyond apoptosis, p53 can enter the mitochondrial matrix and then drive necrosis [30]. Previous studies showed that MRPs selectively act as candidate genes for mitochondrial disease [31], which is associate with the mitochondrial apoptosis $[32,33]$ and the release of mitochondrial Cyt c [34]. As shown in Figure 3B, shMRPS23 did not trigger the release of Cyt c. These data are consistent with the notion that the effect of shMRPS23 is associated with p53 and p21 WAF1/CIP1 activation, but not Cyt c-mediated pathway.

Breast cancer patients are at high risk of recurrence in the form of metastatic disease [3, 35]. EMT is referred to as a key driver in the initiation of the dynamic metastatic process [36]. Consistent with a reversal of the EMT phenotype, down-regulation of
MRPS23 limited the metastasis of the tumour into the surrounding lymph node, rendering the tumour smooth at its edges. During tumour progression, the tumour cells proliferation and metastasis at a distant location relies on cellular attachment to lymph or blood vessels [37]. Our data also revealed that shMRPS23 decreased the mean MVD in junctions between cancerous and non-cancerous tissues, suggesting that shMRPS23-infected cells failed to proliferate and metastasize by blocking angiogenesis. Beyond our expectation, LV-shCtrl group also showed an effect on angiogenesis compared with control group. This is consistent with the notion that lentivirus vector itself depresses angiogenesis [38].

There are several limitations to this study, including the retrospective analysis of correlation between MRPS23 and clinical prognosis in human breast cancer. Furthermore, the true potential of MRPS23 to drive tumour genesis needs to be tested in transgenic mouse models of breast cancer. Further investigations into the molecular mechanisms of MRPS23 in the progression of different breast cancer cells still remain to be elucidated. Our findings suggest that pharmaceutical intervention of shMRPS23 alone or in combination with surgery or radiation therapy might provide a promising strategy to alleviate tumour progression and metastasis.

In summary, we revealed for the first time the biological significance of MRPS23 in breast cancer. Downregulation of MRPS23 results in inhibition of proliferation and metastasis, as well as facilitating apoptosis of breast cancer cells. Additionally, shMRPS23 markedly reduced FDG uptake of the breast cancer cells and impaired angiogenic sprout in vivo. Thus, our findings
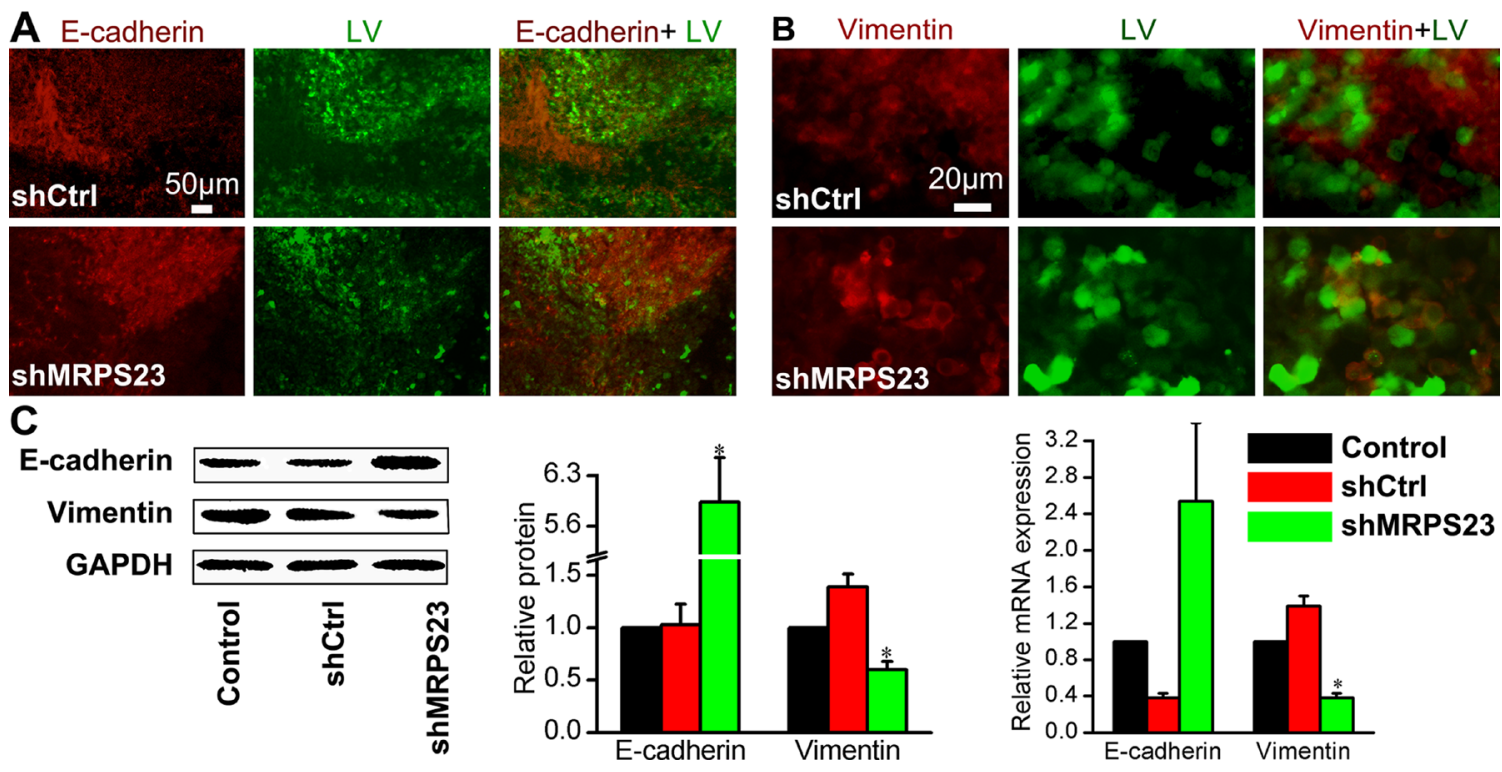

Figure 5: In vivo treatment of shMRPS23 represses breast cancer metastasis by a reversal of EMT. (A, B) Walker256 tumours were immunostained with EMT markers such as E-cadherin (A) or vimentin (B) to examine the effect of shMRPS23 on EMT markers in vivo. (C) Relative protein and mRNA expression of E-cadherin and vimentin as detected by western blot and qPCR analysis in each group. 
suggested that MRPS23 might be a potential therapeutic target to alleviate breast cancer progression and metastasis.

\section{MATERIALS AND METHODS}

\section{Lentivirus vector generation of anti-MRPS23 shRNA vectors}

We generated short hairpin RNA (shRNA) targeting MRPS23 (GenBank accession number NM_001108289.1); the sense strand (5'-GCAGAGTCTTGGAGAAACA-3') was cloned into the lentivirus (LV) expression construct. The vector was successfully co-transfected with LV backbone plasmid into 293T cells using lipofectamine ${ }^{\circledR}$ reagent (Invitrogen, Carlsbad, CA, USA) as reported previously [39]. LV was under the control of the U6 minimal promoter with the gene encoding GFP protein. We named the vector shMRPS23. LV construction and production were completed by Shanghai Gene Chem Co. Ltd., China. The same vector backbone but carrying a nonsilencing sequence (5'-TTCTCCGAACGTGTCACGT-3') was used as a control, and named shCtrl. The average titre of the $\mathrm{LV}$ was at least $5.0 \times 10^{8} \mathrm{TU} / \mathrm{ml}$.

\section{Quantitative real-time PCR assay}

Rat mammary gland carcinoma walker 256 cells in the logarithmic growth phase were plated in 24-well plates at $1 \times$ $10^{5}$ cells, and infected with PBS, LV containing shMRPS23 or LV carrying shCtrl. Total RNA was extracted from cells and tissues using the Trizol reagent (Invitrogen, Carlsbad, CA, USA) and reverse transcribed PCR using the PrimeScript ${ }^{\mathrm{TM}}$ RT Master Mix (Takara, Ohtsu, Japan). Expression levels of targeted genes were analysed by qRT-PCR on a SYBR Green qPCR Master Mix reagent system (Takara, Ohtsu, Japan). The PCR primers used are listed as follows: MRPS23: Forward 5'-TGTCAGCGGTTTGT-GGAG-3'; Reverse 5'-CAGT CTTTGCTTCTCTTACTCGTC-3'; $\beta$-actin: Forward 5'-GG AGATTACTGCCCTGGCTCCTA-3'; Reverse 5'-GACTC ATCGTACTCCTGCTTG-CTG-3'; p53: Forward 5'-GTC GGCTCCGACTATACCACTATC-3'; Reverse 5'-CTCTC TTTGCACT-CCCTGGGGG-3'; $21^{\text {WAFI/CIP1 }}$ : Forward 5'-AG TATGCCGTCGTCTGTTCG-3'; Reverse 5'-TCAAAGTT CCACCGTTCTCG-3'; Cyt c: Forward 5'-AAAGGAG GCAAGCATAAGACTG-3'; Reverse 5'- TGTTCTTGTT GGCATCTGTGT-3'; E-cadherin: Forward 5'-TCTCT TGTCCCTTCCA-CAGC-3'; Reverse 5'-CTCCAGACC CACACCAAAGT-3'; Vimentin: Forward 5'-GGATTT CTCTG-CCTCTTCCA-3'; Reverse 5'-CACCTGTCCGT CTCTGGTTT-3'.

\section{Western blot analysis}

Cell and tissue was homogenized in RIPA lysis buffer (Promega, Madison, US) on ice for $30 \mathrm{~min}$. The concentration of the total protein was measured by the BCA assay (Beyotime, Beijing, China). Western blot analyses were performed as previous study. The antibodies used in this study were as follows: anti-MRPS23 (1:1500; Santa Cruz Biotechnology, Santa Cruz, CA), anti-E-cadherin and anti-Cyt c (1:2000-5000; Abcam, Cambridge, UK), anti-GAPDH, anti-p53, anti-p21 $1^{\mathrm{WAF} / \mathrm{CIP} 1}$, and anti-vimentin (all 1:1000; Cell Signaling Technology).

\section{Cell proliferation and TUNEL stain assay}

Cell viability was measured by Cell Titer 96 AQueous One Solution Cell Assay (MTS, Promega, Madison, US) at $48 \mathrm{~h}$ post transfection. The cells were plated in triplicate at the same initial density. The terminal deoxynucleotidyl transferase dUTP nick-end labelling (TUNEL, KeyGEN Biotec, Nanjing, China) assay was performed to assess the cellular apoptosis $48 \mathrm{~h}$ post $\mathrm{LV}$ shRNA infection. Confocal laser scanning microscopy images of Walker 256 carcinoma cells, triple labelled with EGFP (green pseudocolour), TRITC-TUNEL (red pseudocolour) and DAPI (blue pseudocolor) was used to analyze apoptotic index as previously described [40].

\section{Tumour and animal model}

For in vivo experiments, ascites Walker 256 tumour cells were prepared and suspended in $10 \mu \mathrm{l}$ isotonic saline containing $10^{5}$ cells. For implantation, in brief, fifteen Specified pathogen free female Sprague Dawley (SD) rats (200-250 g) were obtained and housed in the Experimental Animal Center of Hubei University of Medicine (Shiyan, China). For implantation, in brief, animals were anesthetized with isoflurane ( $3 \%$ induction, $2 \%$ maintenance), and tumour cells respectively infected with LV expressing shRNA control (shCtrl), LV expressing shMRPS23 (shMRPS23) or PBS (Control) were injected into the bone cavity as previous described $[41,42]$. After surgery, rats were placed on a warm pad for recovery, randomly divided into three groups $(n=5)$, and allowed access to food and water ad libitum. All animal experimental protocols were approved by the Institutional Animal Care in the Hubei University of Medicine Taihe Hospital, and the experiments were carried out in accordance with the approved guidelines.

\section{In vivo $\mathrm{PET} / \mathrm{CT}$ imaging}

To assess the metabolism of tumour, four rats of each group were prepared for PET imaging 4 weeks after tumour cell injection. The animals were warmed and anesthetized throughout the entire imaging procedure, and then injected with $0.1 \mathrm{mCi}^{18} \mathrm{~F}-\mathrm{FDG}$ via a tail vein. After $1 \mathrm{~h}$ of unconscious uptake, images were acquired on a micro-PET/CT scanner (Siemens Inc., Erlangen, Germany) in the previous study [43]. The quantitative evaluation was performed by drawing $3 \mathrm{D}$ region of interests (ROIs). Maximum standardized uptake values (SUVmax) normalized for body weight and total radioactivity and metabolic tumour volume were calculated. 
After the PET scanning, the animals were sacrificed with an overdose of isoflurane on the next day and tumour tissues were retrieved for the following analysis.

\section{Immunofluorescence and immunohistochemistry assay}

Walker256 cells were cultured and infected with the indicated LV for $48 \mathrm{~h}$, then stained with $50 \mu \mathrm{M}$ MitoTracker Deep Red FM (Invitrogen, Carlsbad, CA) at $37^{\circ} \mathrm{C}$ for $30 \mathrm{~min}$. This was followed by incubation with MRPS23 primary antibody and with Donkey anti-goat Alexa Fluro 405 (1:400; Abcam, Cambridge, UK) as previously reported [44]. In fresh-frozen tumour sections, tumours were fixed in $4 \%$ paraformaldehyde, embedded with $\mathrm{OCT}$, frozen at $-20^{\circ} \mathrm{C}$, cut into slices with $20 \mu \mathrm{m}$ thickness. Protein antigens were detected by staining with the indicated primary antibodies overnight at $4^{\circ} \mathrm{C}$, followed by incubation with a secondary antibody at $25^{\circ} \mathrm{C}$ for 30 min. Donkey anti-rabbit Alexa Fluor-568 and Donkey anti-mouse Alexa Fluor-405 antibodies (1:400, Abcam, Cambridge, UK) were used as secondary antibodies. Immunofluorescent labelling tissues were observed under a Leica fluorescent inverted microscope. The microvessel density (MVD) was assessed by CD34 Ab staining with the method described in the previous study [20,45].

\section{Statistical data analyses}

The results were expressed as means $\pm \mathrm{SD}$. All statistical analysis was performed by SPSS 17.0 software. One-way ANOVA was used to compare the differences between groups. ${ }^{*} p<0.05$ was considered statistically significant.

\section{ACKNOWLEDGMENTS}

This study was supported by the National Natural Science Foundation of China (grant number 81401447), Science and Technology Development Foundation of Shiyan City (NO. 16Y16) and the Key Discipline Project of Hubei University of Medicine. We also thank Ruoshi Shi and Wanjun Yao for assistance with cell culture and animal model in our laboratory.

\section{CONFLICTS OF INTEREST}

Competing financial interests: All the authors declare that they have no competing interest.

\section{REFERENCES}

1. Stewart B, Wild CP. World cancer report 2014. World. 2016.

2. Fan L, Strasser-Weippl K, Li JJ, Finkelstein DM, Yu KD, Chen WQ, Shao ZM, Goss PE. Breast cancer in China. The lancet oncology. 2014; 15:e279-e89.
3. Early Breast Cancer Trialists' Collaborative Group (EBCTCG). Adjuvant bisphosphonate treatment in early breast cancer: meta-analyses of individual patient data from randomised trials. The Lancet. 2015; 386:1353-61.

4. Costelloe CM, Rohren EM, Madewell JE, Hamaoka T, Theriault RL, Yu TK, Lewis VO, Ma J, Stafford RJ, Tari AM. Imaging bone metastases in breast cancer: techniques and recommendations for diagnosis. The lancet oncology. 2009; 10:606-14.

5. Gatza ML, Silva GO, Parker JS, Fan C, Perou CM. An integrated genomics approach identifies drivers of proliferation in luminal-subtype human breast cancer. Nat Genet. 2014; 46:1051-9.

6. Dogan AL. (2016). Biology and Genetics of Breast Cancer. Breast Dis: Springer), pp. 145-60.

7. Zhang Q, Wang JP. A Bayesian network approach for modeling mixed features in TCGA ovarian cancer data. bioRxiv. 2015:033332.

8. Staub E, Grone J, Mennerich D, Ropcke S, Klamann I, Hinzmann B, Castanos-Velez E, Mann B, Pilarsky C, Brummendorf T, Weber B, Buhr HJ, Rosenthal A. A genome-wide map of aberrantly expressed chromosomal islands in colorectal cancer. Mol Cancer. 2006; 5:37.

9. Bin L, Zhang YL. Identification of up-regulated genes in human uterine leiomyoma by suppression subtractive hybridization. Cell Res. 2002; 12:215-21.

10. Kurokawa Y, Matoba R, Nakamori S, Takemasa I, Nagano H, Dono K, Umeshita K, Sakon M, Monden M, Kato K. PCR-array gene expression profiling of hepatocellular carcinoma. J Exp Clin Cancer Res. 2004; 23:135-42.

11. Kristensen G, Lyng H, Svendsrud D, Holm R, Knutstad K, Brovig R, Sundfoer K, Oksefjell H, Stokke T. (2006). Molecular markers for lymph node involvement in locally advanced cervical carcinomas. ASCO Annual Meeting Proceedings, pp. 5049.

12. Lyng H, Brovig RS, Svendsrud DH, Holm R, Kaalhus O, Knutstad K, Oksefjell H, Sundfor K, Kristensen GB, Stokke T. Gene expressions and copy numbers associated with metastatic phenotypes of uterine cervical cancer. BMC Genomics. 2006; 7:268.

13. Shamanna RA, Hoque M, Pe'ery T, Mathews MB. Induction of p53, p21 and apoptosis by silencing the NF90/NF45 complex in human papilloma virus-transformed cervical carcinoma cells. Oncogene. 2013; 32:5176-85.

14. Chen YC, Chang MY, Shiau AL, Yo YT, Wu CL. Mitochondrial ribosomal protein S36 delays cell cycle progression in association with p53 modification and p21(WAF1/CIP1) expression. J Cell Biochem. 2007; 100:981-90.

15. Yoo YA, Kim MJ, Park JK, Chung YM, Lee JH, Chi SG, Kim JS, Yoo YD. Mitochondrial ribosomal protein L41 suppresses cell growth in association with p53 and p27Kip1. Mol Cell Biol. 2005; 25:6603-16. 
16. Kim TW, Kim B, Kim JH, Kang S, Park SB, Jeong G, Kang HS, Kim SJ. Nuclear-encoded mitochondrial MTO1 and MRPL41 are regulated in an opposite epigenetic mode based on estrogen receptor status in breast cancer. BMC Cancer. 2013; 13: 502.

17. Wang X, Peralta S, Moraes CT. Mitochondrial alterations during carcinogenesis: a review of metabolic transformation and targets for anticancer treatments. Adv Cancer Res. 2013; 119:127-60.

18. Suzuki H, Ito R, Ikeda K, Tamura TA. TATA-binding protein (TBP)-like protein is required for p53-dependent transcriptional activation of upstream promoter of $\mathrm{p} 21 \mathrm{Wafl} /$ Cip1 gene. J Biol Chem. 2012; 287:19792-803.

19. Galanos P, Vougas K, Walter D, Polyzos A, MayaMendoza A, Haagensen EJ, Kokkalis A, Roumelioti FM, Gagos S, Tzetis M. Chronic p53-independent p21 expression causes genomic instability by deregulating replication licensing. Nat Cell Biol. 2016.

20. Masiero M, Simoes FC, Han HD, Snell C, Peterkin T, Bridges E, Mangala LS, Wu SY, Pradeep S, Li D, Han C, Dalton H, Lopez-Berestein G, et al. A core human primary tumor angiogenesis signature identifies the endothelial orphan receptor ELTD1 as a key regulator of angiogenesis. Cancer Cell. 2013; 24:229-41.

21. Imani S, Hosseinifard H, Cheng J, Wei C, Fu J. Prognostic Value of EMT-inducing Transcription Factors (EMT-TFs) in Metastatic Breast Cancer: A Systematic Review and Metaanalysis. Sci Rep. 2016; 6:28587.

22. Lamouille S, Xu J, Derynck R. Molecular mechanisms of epithelial-mesenchymal transition. Nat Rev Mol Cell Biol. 2014; 15:178-96.

23. Christian BE, Spremulli LL. Mechanism of protein biosynthesis in mammalian mitochondria. Biochimica et Biophysica Acta (BBA)-Gene Regulatory Mechanisms. 2012; 1819:1035-54.

24. Dennerlein S, Rozanska A, Wydro M, ChrzanowskaLightowlers ZM, Lightowlers RN. Human ERAL1 is a mitochondrial RNA chaperone involved in the assembly of the 28S small mitochondrial ribosomal subunit. Biochemical Journal. 2010; 430:551-8.

25. Richter U, Lahtinen T, Marttinen P, Myohanen M, Greco D, Cannino G, Jacobs HT, Lietzen N, Nyman TA, Battersby BJ. A mitochondrial ribosomal and RNA decay pathway blocks cell proliferation. Curr Biol. 2013; 23:535-41.

26. Tian Y, Qin L, Qiu H, Shi D, Sun R, Li W, Liu T, Wang J, $\mathrm{Xu}$ T, Guo W. RPS3 regulates melanoma cell growth and apoptosis by targeting Cyto $\mathrm{C} / \mathrm{Ca} 2+/ \mathrm{MICU} 1$ dependent mitochondrial signaling. Oncotarget. 2015; 6:29614. https:// doi.org/10.18632/oncotarget.4868.

27. Russo A, Esposito D, Catillo M, Pietropaolo C, Crescenzi E, Russo G. Human rpL3 induces G(1)/S arrest or apoptosis by modulating p21 (waf1/cip1) levels in a p53-independent manner. Cell Cycle. 2013; 12:76-87.

28. Russo A, Pagliara V, Albano F, Esposito D, Sagar V, Loreni F, Irace C, Santamaria R, Russo G. Regulatory role of rpL3 in cell response to nucleolar stress induced by Act D in tumor cells lacking functional p53. Cell Cycle. 2016; 15:41-51.

29. Kim MJ, Yoo YA, Kim HJ, Kang S, Kim YG, Kim JS, Yoo YD. Mitochondrial ribosomal protein L41 mediates serum starvation-induced cell-cycle arrest through an increase of p21(WAF1/CIP1). Biochem Biophys Res Commun. 2005; 338:1179-84.

30. Vaseva AV, Marchenko ND, Ji K, Tsirka SE, Holzmann S, Moll UM. p53 opens the mitochondrial permeability transition pore to trigger necrosis. Cell. 2012; 149:1536-48.

31. Williams CC, Jan CH, Weissman JS. Targeting and plasticity of mitochondrial proteins revealed by proximityspecific ribosome profiling. Science. 2014; 346:748-51.

32. Modjtahedi N, Tokatlidis K, Dessen P, Kroemer G. Mitochondrial proteins containing coiled-coil-helix-coiledcoil-helix $(\mathrm{CHCH})$ Domains in Health and Disease. Trends Biochem Sci. 2016; 41:245-60.

33. Zhang X, Gao X, Coots RA, Conn CS, Liu B, Qian SB. Translational control of the cytosolic stress response by mitochondrial ribosomal protein L18. Nat Struct Mol Biol. 2015; 22:404-10.

34. Antonicka H, Shoubridge EA. Mitochondrial RNA granules are centers for posttranscriptional RNA processing and ribosome biogenesis. Cell reports. 2015; 10:920-32.

35. Kovacheva M, Zepp M, Berger SM, Berger MR. Sustained conditional knockdown reveals intracellular bone sialoprotein as essential for breast cancer skeletal metastasis. Oncotarget. 2014; 5:5510-22. https://doi. org/10.18632/oncotarget.2132.

36. Lamouille S, Xu J, Derynck R. Molecular mechanisms of epithelial-mesenchymal transition. Nature reviews Molecular cell biology. 2014; 15:178.

37. Croci DO, Cerliani JP, Dalotto MT, Mendez SP, Mascanfroni ID, Dergan DS, Toscano MA, Caramelo JJ, Garcia JJ, Ouyang J, Mesri EA, Junttila MR, Bais C, et al. Glycosylation-dependent lectin-receptor interactions preserve angiogenesis in anti-VEGF refractory tumors. Cell. 2014; 156:744-58.

38. Seth P. Vector-mediated cancer gene therapy: An overview. Cancer Biol Ther. 2014; 4:512-7.

39. He B, Xiao YF, Tang B, Wu YY, Hu CJ, Xie R, Yang X, Yu ST, Dong H, Zhao XY, Li JL, Yang SM. hTERT mediates gastric cancer metastasis partially through the indirect targeting of ITGB1 by microRNA-29a. Sci Rep. 2016; 6:21955.

40. Pei Z, Du X, Song Y, Fan L, Li F, Gao Y, Wu R, Chen Y, Li W, Zhou H, Yang Y, Zeng J. Down-regulation of lncRNA CASC2 promotes cell proliferation and metastasis of bladder cancer by activation of the Wnt/ $\beta$-catenin signaling pathway. Oncotarget. 2017; 8:18145. https://doi. org/10.18632/oncotarget.15210.

41. Luo J, Huang X, Li Y, Li Y, Xu X, Gao Y, Shi R, Yao W, Liu J, Ke C. GPR30 disrupts the balance of GABAergic 
and glutamatergic transmission in the spinal cord driving to the development of bone cancer pain. Oncotarget. 2016; 7:73462-73472. https://doi.org/10.18632/oncotarget.11867.

42. Ke C, Gao F, Tian X, Li C, Shi D, He W, Tian Y. Slit2/ Robol mediation of synaptic plasticity contributes to bone cancer pain. Molecular neurobiology. 2016:1-13.

43. Pei Z, Lan X, Cheng Z, Qin C, Xia X, Yuan H, Ding Z, Zhang Y. Multimodality molecular imaging to monitor transplanted stem cells for the treatment of ischemic heart disease. PloS one. 2014; 9:e90543.
44. Zhuang J, Wang PY, Huang X, Chen X, Kang JG, Hwang PM. Mitochondrial disulfide relay mediates translocation of p53 and partitions its subcellular activity. Proceedings of the National Academy of Sciences. 2013; 110:17356-61.

45. Al-Hilal TA, Chung SW, Choi JU, Alam F, Park J, Kim SW, Kim SY, Ahsan F, Kim IS, Byun Y. Targeting prion-like protein doppel selectively suppresses tumor angiogenesis. The Journal of clinical investigation. 2016; 126:1251-66. 